\title{
Patient- or Participant-Generated Registries
}




\section{Research White Paper}

\section{Patient- or Participant-Generated Registries}

\section{Addendum to Registries for Evaluating Patient Outcomes: A User's Guide, Third Edition}

\section{Prepared for:}

Agency for Healthcare Research and Quality

U.S. Department of Health and Human Services

5600 Fishers Lane

Rockville, MD 20857

www.ahrq.gov

Contract No. 290-2014-00004-C

Prepared by:

L\&M Policy Research, LLC

Washington, DC

Authors (alphabetical):

Sharon Terry, M.A. (Lead)

Genetic Alliance

Deborah Runkle

American Association for the Advancement of Science

Paul Wicks, Ph.D.

PatientsLikeMe

AHRQ Publication No. 17(18)-EHC017-EF

February 2018 
The Effective Health Care Program of the Agency for Healthcare Research and Quality (AHRQ) conducts and supports research focused on the outcomes, effectiveness, comparative clinical effectiveness, and appropriateness of pharmaceuticals, devices, and health care services. More information on the Effective Health Care Program can be found at www.effectivehealthcare.ahrq.gov.

This report is based on research conducted by L\&M Policy Research, LLC, with partners OM1 and IQVIA, under contract to the Agency for Healthcare Research and Quality, Rockville, MD (Contract No. 290-2014-00004-C). The findings and conclusions in this document are those of the authors, who are responsible for its contents; the findings and conclusions do not necessarily represent the views of AHRQ. Therefore, no statement in this report should be construed as an official position of AHRQ or of the U.S. Department of Health and Human Services.

\section{None of the authors have any affiliations or financial involvement that conflicts with the material presented in this report.}

This report may be used and reprinted without permission except those copyrighted materials that are clearly noted in the report. Further reproduction of those copyrighted materials is prohibited without the express permission of copyright holders.

Persons using assistive technology may not be able to fully access information in this report. For assistance contact EPC@ahrq.hhs.gov.

Suggested citation: Terry S, Runkle D, Wicks P. Patient- or Participant-Generated Registries. White Paper, addendum to Registries for Evaluating Patient Outcomes: A User's Guide, Third Edition. (Prepared by L\&M Policy Research, LLC, under Contract No. 290-2014-00004-C.) AHRQ Publication No. 17(18)-EHC017-EF. Rockville, MD: Agency for Healthcare Research and Quality; February 2018. www.effectivehealthcare.ahrq.gov. DOI: https://doi.org/10.23970/AHRQREGISTRIESGENERATED. 


\section{Reviewers (alphabetical):}

Alan Balch, Ph.D.

Patient Advocate Foundation

Jennifer B. Christian, Pharm.D., M.P.H., Ph.D., FISPE

Vice President, Clinical Evidence

Center for Advanced Evidence Generation

IQVIA

Nancy A. Dreyer, Ph.D., M.P.H., FISPE

Chief of Scientific Affairs \& Head Center

for Advanced Evidence Generation

IQVIA

Richard E. Gliklich, M.D.

Chief Executive Officer and Chairman

OM1

Lisa Heral, R.N.B.A.

Fibrous Dysplasia Foundation

Michelle B. Leavy, M.P.H.

OM1

Paul Lipkin, M.D.

Interactive Autism Network 


\section{Contents}

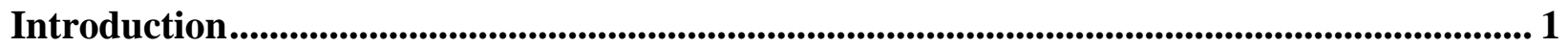

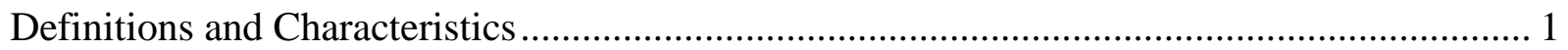

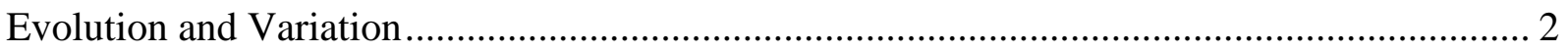

Purpose and Goals of Patient- or Participant-Generated Registries ............................................ 3

Give Voice to People Living With the Condition or Issue .............................................................. 3

Natural History of Disease.................................................................................................. 3

Hypothesis Generation ......................................................................................................... 4

Patient-Reported Outcomes …………………………....................................................... 5

Identification of Participants and Other Support for Clinical Trials and Cohort Studies........... 6

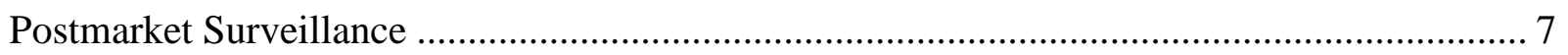

Visibility of Registry Data and Communicating Research Findings ........................................... 7

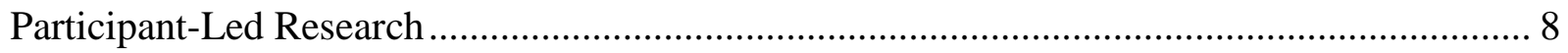

Support of Development of Treatment Guidelines ................................................................. 10

Planning and Design Considerations ..................................................................................................... 10

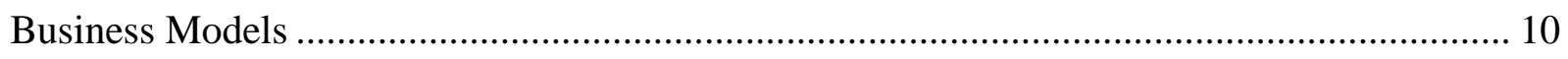

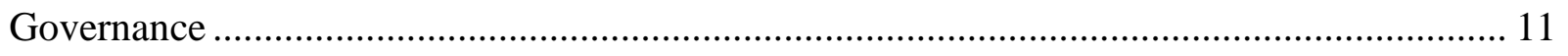

Ethics and Informed Consent................................................................................................... 11

Challenges and Limitations...................................................................................................................... 12

Participant Privacy and Confidentiality .............................................................................. 12

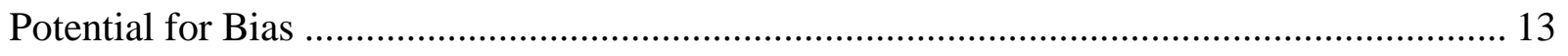

Validation of Participant Population.................................................................................... 13

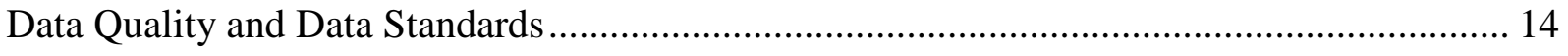

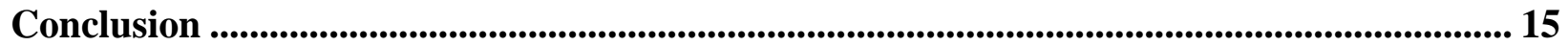

References ..................................................................................................................................................... 16 


\section{Introduction}

Patient registries can provide important, real-world evidence to inform health decisions made by patients, consumers, providers, as well as regulatory agencies and payers. Many patient registries are sponsored by professional associations, academic institutions, healthcare organizations, or pharmaceutical and medical device companies. Within the past 20 years, patient- or participantgenerated registries have emerged as a new form of registry, are an important tool for generating information of direct relevance to the patient community. These registries, created, managed, and/or governed directly by the participants themselves, are an important tool for generating information of direct relevance to the patient community.

\section{Definitions and Characteristics}

The defining characteristics of patient- or participant-generated registries (PGRs) as described here are that they are created by patients, or by people who may not describe themselves primarily as patients. As such, these registries are often termed a "patient-generated registry," a "patient-powered registry," or a "participant-generated registry.” The most appropriate terminology for these registries is the subject of some debate since the term 'patient' itself seemingly limits the discussion to individuals who are currently receiving medical treatment. Further, describing people with a common characteristic as "patients" may suggest a notion of information asymmetry, in which the patient has less knowledge than a medical professional. In reality, many individuals who participate in registries, such as those with chronic, lifelong illnesses, do not define themselves as patients except when they are in the specific position of being in a clinical care setting. In addition, some individuals create and manage registries to study health-related questions to some characteristic they have in common, but are not defined as patients. Consider, for example, a registry designed to track the long-term health-related outcomes of men who were recently released from prison, or another that considers the health issues for people who are lesbian, gay, bisexual,and transgender. ${ }^{1}$

In this paper, the authors have used the abbreviation "PGR", which might represent either patients or participants in order to recognize the identity and experiences of registry participants beyond the focus on their medical care and role as patients. The terms "participants" and "patients" will both be used to refer to registry enrollees, depending on the context, in this chapter. The intentionally broad language used here merits review and reconsideration in coming years as this field continues to evolve.

As the field of PGRs is rapidly changing and expanding, it is helpful to consider PGRs through the lens of defining characteristics - as in, what are the primary features that distinguish the PGR from the investigator/sponsor-generated (i.e., traditional) registry. For PGRs, such defining characteristics may include some or all of the following:

- Founded, owned, and managed by those who participate in the registry, whether or not they are associated with disease advocacy groups;

- Focus on the needs and goals of the registry participants as the first principle; 
- Employ social networks and/or community-based engagement to recruit participants; and/or

- Collect participant-reported health data in a structured and/or unstructured format, in addition to data generated during the healthcare encounter.

It is also important to emphasize that PGRs do not only collect participant-reported data. Many incorporate data from the participants' health records, both paper and electronic, clinical visits, and, in some cases, collect biological samples. Moreover, PGRs may use any type of retrospective and/or prospective cohort study design used for other types of registries, including direct-to-patient designs and other patient-centric designs that are discussed in the eBook Chapter Direct-to-Patient Registry and Other Patient-Centric Designs. ${ }^{2}$ Many of these characteristics may also apply to some investigator/sponsor-generated registries, but they are of particular importance for PGRs.

\section{Evolution and Variation}

The phenomenon of individuals affected by diseases establishing registries is more than 20 years old. ${ }^{3}$ However, PGRs have benefited from recent advances in social network technologies. PGRs generally rely heavily on the combination of Moore's Law, ${ }^{4}$ which predicts that overall processing power for computers will double every two years, and Metcalfe's Law, which predicts the value of networks to increase in proportion to the size of the those connected to the network. ${ }^{5,6}$ The confluence of these factors has created an environment in which PGRs have flourished, albeit with wide variation.

Wide variation in PGRs is evident in the results of a 2014 survey conducted by the American Association for the Advancement of Science (AAAS) with funding from the Agency for Healthcare Research and Quality (AHRQ). ${ }^{7}$ While the survey represented a very small sample of PGRs, it offers evidence that PGRs are not organized around any single attribute. Of the 30 responding PGRs, the majority were initiated by individuals affected by a disease or condition and/or an advocacy organization dedicated to a condition. In some cases, a family member who is also a scientist or clinician initiated the registry. As an example, Leslie Gordon and Scott Berns, pediatricians whose son suffered from Hutchison-Gilford progeria syndrome, which is a very rare genetic disorder that dramatically accelerates aging, ${ }^{8}$ founded both a support organization, the Progeria Research Foundation, and a registry for the condition, the International Progeria Registry. ${ }^{9}$ The registry has not only provided direct insight into the most appropriate care for children with this rare condition, but also is a catalyst for translational research. In fact, study of the cellular mechanisms involved in progeria have led to two treatments for the condition. ${ }^{10}$ In addition, the apparent premature aging of progeria patients is providing opportunities to better understand mechanisms of the normal aging process and cardiovascular disease, with the possibility of developing new interventions for these processes.

The PGRs responding to the survey also differ in how they are managed. About half of the registries in the sample are managed by advocacy organizations, with the others managed by the participants themselves, with or without the collaboration of health care providers/researchers. 
Funding comes from a variety of sources as well, with private donors providing funding for about half of the registries that replied to the AAAS survey, the Federal government providing funding for 23 percent of the responding registries, and advocacy organizations and participants providing funds for the remainder. While some PGRs may be organized as for-profit ventures, AAAS reported that most registries responding to the survey were nonprofit organizations. Taken as a whole, the key differentiator of PGRs from traditional registries is that they are founded, managed and/or governed by individuals with a lived experience of the condition under study.

A second key differentiator is the role of the participant in the governance of the registry. The North American Research Committee on Multiple Sclerosis (NARCOMS) registry collects participant-reported data on more than 37,000 participants living with multiple sclerosis (MS) and considers itself to be "participant-driven." However, the registry was developed by academics and focuses on supporting the research work of MS investigators. ${ }^{11}$ By contrast, iConquerMS $^{12}$ is an online platform that gathers similar participant-reported data but includes participants in the governance structure and development of the research and was founded by a participant group (the Accelerated Cure Project), thus, meeting several of the key characteristics of a PGR.

Collection of patient-reported outcome (PRO) measures used to be considered a differentiator for PGRs in comparison to data collected by traditional registries, but increasingly, traditional registries are also collecting participant-reported data, often in the form of patient-reported exposures and PRO measures. ${ }^{13,14}$

\section{Purpose and Goals of Patient- or Participant-Generated Registries}

Like traditional investigator/sponsor-generated registries, PGRs have many purposes and are often designed to address multiple objectives. PGRs are likely to be established when the issues, concerns, or disease itself are not a priority for traditional sponsors of registries. Accordingly, one of the goals of PGRs may be to engage enough patients or participants to attract the interest of scientists and/or industry to embark on development of new therapies. These and other goals of PGRs are summarized further here.

\section{Give Voice to People Living With the Condition or Issue}

Many PGRs offer social media opportunities to enable participants to share or discuss details of their condition and life experiences. The social media tools, which support development of a patient community, are not typically found in traditional patient registries and represent a unique aspect of PGRs.

\section{Natural History of Disease}

PGRs have been especially successful and important in establishing the natural history of a disease. Advocacy organizations focused on a disease or condition are able to track natural history across many dimensions over many years, which usually goes beyond the commitment of a single sponsor, funder or investigator team. Moreover, these organizations remain involved in 
participants' lives in ways that are outside the scope of traditional registries. In fact, research groups that have either finished a project or lost funding, have sometimes turned over registry data or biospecimens to advocacy organizations to act as the stewards of these data and samples.

An excellent example of a longstanding, successful registry with a focus on natural history of disease is the Cystic Fibrosis Foundation (CFF) Patient Registry. The registry, which began collecting data over 50 years ago, has documented the disease course, helped establish treatment guidelines and best practices, and contributed to the creation of a robust therapy development pipeline. For 50 years, the CFF Patient Registry has recorded the increase in expected life span for cystic fibrosis (CF) patients from as young as five years old to about 50 years old. ${ }^{15,16}$

Another organization, Parent Project Muscular Dystrophy (PPMD), a nonprofit organization focused on finding a cure for Duchenne's muscular dystrophy (DMD), has catapulted research forward because of its robust registry, DuchenneConnect. While providing over \$45 million in direct funding for development of new therapies in DMD, PPMD also makes continual use of its registry for natural history, biomarkers, and historic controls. ${ }^{17,18}$ In some cases, advocacy organizations for rare conditions cannot afford the infrastructure necessary for establishing a registry. In 2003, for that reason, and to conserve resources for all disease advocacy organizations, the Genetic Alliance established the Genetic Alliance Registry and BioBank to support research across a range of conditions using a cooperative collaborative model to offer infrastructure, template documents, and technical assistance to any nonprofit in need of registry or bio-banking services. At the present time it contains more than 30,000 samples of a variety of tissue types from DNA to cell lines to whole body harvests across about eight contributing registries. $^{19}$

PGRs can also be useful in terms of providing information about the natural history of disease that can be used for comparative purposes in drug development. Consider the challenge of obtaining regulatory approvals for new indications of marketed products, particularly for rare conditions. A PGR can focus on collecting the data needed to support repurposing of the drug. ${ }^{20}$ For example, the Progeria Research Foundation used its registry to build the necessary cohort to reposition Lonafarnib to reverse instability of nuclear structure due to the genetic mutation of the LMNA gene. The drug, called Sarasar by its manufacturer Schering-Plough, was tested in Phase I/II/III clinical trials for a variety of cancers. The Progeria Research Foundation used its registry, through which they had conducted a natural history study, as the "control" needed in the Phase I and II trials. $^{21}$

\section{Hypothesis Generation}

One area of strength for PGRs is to catalyze hypothesis generation that can then be followed-up by more rigorous studies, rather than engage solely in hypothesis testing. In this context, the aim is to quickly evaluate signals that might be challenging to study through traditional means or with traditional funding sources. In one example, researchers from Oxford University sought to test the hypothesis that right-handed participants with amyotrophic lateral sclerosis (ALS) were more likely to experience their first symptoms on their dominant side. A traditional approach might have recruited a consecutive case series from a major referral center; however, analysis of 
the statistical power required to conduct an informative study suggested that several hundred participants would be needed, requiring several years' worth of data collection for such a rare disorder. By surveying participants willing to be contacted for research studies on PatientsLikeMe, 502 participants responded and were available for analysis in four weeks. ${ }^{22}$ The study rapidly indicated that among those with an arm onset there was 64-percent concordance with dominance and only 55-percent concordance with leg onset. While the sample from the PGR was subject to limitations and biases, the analysis provided rapid proof of a concept that was subsequently validated and replicated by clinical studies. The analysis also served as a basis for collecting more detailed neuroimaging work. ${ }^{22,23}$

Another example of hypothesis-generation is PXE International, an advocacy organization founded by parents of children affected by pseudoxanthoma elasticum (PXE). This organization was developed to promote and fund research that will lead to better management and interventions for people living with PXE, a rare genetic disease that affects the eyes, skin, cardiovascular and gastrointestinal systems. Indeed, the PXE International Registry has produced a number of hypotheses that have progressed to more focused clinical studies, ultimately leading to changes in clinical care. For example, several individuals reporting later-onset PXE after a liver transplant precipitated the hypothesis that PXE is a metabolic disease, and further research confirmed this hypothesis. ${ }^{24}$ The PXE International Registry also led to discoveries about pregnancy, mammography, and eye treatments in affected individuals, thus changing practice guidelines. $^{25-27}$

\section{Patient-Reported Outcomes}

PROs reflect the status of a patient's health condition that comes directly from the patient, without interpretation of the patient's response by a clinician or any other individual. ${ }^{28}$ They are often assessed through structured questionnaires that directly survey patients or other participants about their experiences of symptoms or impact of disease upon their quality of life, and are increasingly used as study endpoints because of their clinical relevance or as catalysts to initiate more in-depth clinical and behavioral research. PGRs can provide an opportunity for participants building on one another's experiences to measure what matters to them and create new PRO tools that can be used by other researchers in future studies. In one example, while completing the widely used PRO for ALS, the Revised ALS Functional Rating Scale (ALSFRS-R), ${ }^{29}$ a person living with ALS was irritated to find that despite being able to participate in family life and the running of her household, the scale scored her as "zero". ${ }^{30}$ In response to this apparent denial of her current capabilities, she worked to engage with other severely affected ALS participants to develop a new suite of items that extended the "floor level" of the ALSFRS-R to tap previously unmeasured areas of function, such as communicating through facial expression rather than through voice or handwriting, or through use of assistive technology. These items formed the ALSFRS Extension Items (ALSFRS-EX), which have been translated into other languages and form the measure of choice in the Veterans Affairs' (VA) Biorepository Brain Bank. ${ }^{31-33}$

There are many PRO tools. Some are validated instruments and others are not validated. There is interest from the United States Food and Drug Administration (FDA) to require that PRO 
instruments meet rigorous criteria demanded in order to achieve broad use in research studies. ${ }^{34}$ Therefore, another potential use of PGRs is facilitating the development of new PROs through each of the required stages of instrument development and validation required for labeling. ${ }^{28}$ Other purposes include concept elicitation, cognitive debriefing, psychometric testing, test-retest, and measurement of changes over time to assess sensitivity. One initiative, the Open Research Exchange (ORE), funded by the Robert Wood Johnson Foundation, allows researchers to more quickly proceed through each step of the PRO validation process with volunteers recruited from PatientsLikeMe. Rather than the months or years needed to go through each stage of development, gathering the data electronically can take only days or weeks. For example, a French group was able to gather data from over 600 participants living with chronic illness to validate an English version of their Treatment Burden Questionnaire (TBQ) in only a month. ${ }^{35,36}$

A commentary piece co-authored with an FDA endpoint reviewer lays out the hurdles that need to be overcome for such systems to be credible. ${ }^{36}$ Researchers developing PROs must establish that participants are who they say they are, which can be accomplished by matching their records to claims data or electronic health records (EHRs); ${ }^{37}$ the data quality must be comparable to existing methods (as early studies are indicating); ${ }^{38}$ and the population must be shown to be generalizable, which is achievable with a large sample. PROs developed with the help of PGR participants may address these hurdles by being scientifically rigorous, broadly generalizable to a wider range of patients with the disease of interest, and responsive to a community's needs while also alleviating some of the logistical challenges of PRO development for researchers.

\section{Identification of Participants and Other Support for Clinical Trials and Cohort Studies}

Registries may be designed with the goal of providing an identified source population of participants with a condition who might be enrolled in observational studies and/or clinical trials. Data in the registry may be used to identify participants who meet the inclusion criteria for a planned trial, to assess the feasibility of enrolling the intended sample size per protocol criteria, to determine desirable geographic locations of enrollment centers, and to facilitate trial enrollment by sharing information about the trial with participants who meet the inclusion criteria.

PGRs may also be designed to facilitate future clinical trials. As an example, the Batten Disease Support and Research Association accelerated research undertaken by BioMarin as they conducted a clinical trial of enzyme replacement therapy for CLN2 Batten (Late-Infantile Neuronal Ceroid Lipofuscinosis Type 2) by providing historical control data. ${ }^{39}$ Regulatory authorities determined the historical control data to be sufficient for understanding the natural history and course of the disease in untreated patients, and allowed all children enrolled in the trial to receive the active therapy. ${ }^{40}$

PGRs also initiate and conduct trials themselves. In addition, for those not taking such a committed step, they might provide input before, during, and after clinical studies are designed and implemented. Before a study starts enrolling participants, there is increasing recognition that co-creation of the design and conduct of research studies can better ensure the results are relevant and meaningful to participants. The requirement for patient and participant engagement 
as part of research study design and conduct is mandated for studies funded by the PatientCentered Outcomes Research Institute (PCORI). ${ }^{41,42}$

In early 2015, during an announcement of the Precision Medicine Initiative, President Obama declared that people would be partners in clinical studies. ${ }^{43,44}$ The FDA's Patient-Focused Drug Development Initiative shares this goal as well and directly seeks patient and patient advocate input through a series of disease-specific workshops intended to inform development of new therapies and acceptability of clinical trial designs. ${ }^{45}$ Through this program, preferences collected by PPMD have helped to inform the FDA's benefit-risk assessment of emerging DMD therapies. ${ }^{46}$

Once a study protocol has been developed, awareness campaigns targeted to eligible patients have been useful in speeding recruitment into clinical trials. ${ }^{47}$ Some recruitment tools developed in PGRs such as the Platform for Engaging Everyone Responsibly (PEER, one version known as Reg4All, formerly known as TrialsFinder), ${ }^{48}$ and the Michael J. Fox Foundation's Fox Trial Finder, ${ }^{49}$ provide potential trial participants with curated lists of clinical trials to aid recruitment, while PatientsLikeMe ${ }^{50}$ and many patient advocacy organizations have tools that allow people to search current listings of trials on ClinicalTrials.gov.

PGRs may also establish collaborations with organizations conducting clinical trials of new interventions. PPMD uses its registry, DuchenneConnect, to engage in clinical trials and device trials as well. At the end of 2014, the Foundation announced a collaboration with Solid Ventures to engage SRI International in the research and development of the "Solid Suit," a soft, wearable assistive device for people with DMD.

\section{Postmarket Surveillance}

In the setting of postmarket surveillance, the Empowered Patient Coalition in the United States is an example of committed patients creating a registry of perceived adverse events in order to empower people to take control of their own medical treatments and safety. ${ }^{51}$ While direct attribution of these adverse events to medical errors may not be possible or appropriate, a recent article in the British Medical Journal describes the goal as to "relay to healthcare providers and administrators a first-hand quantitative and qualitative view of the impact that adverse medical events has on patients and their families”, and notes that other studies of medical errors, being clinician-led, exclude the voice of the patient and may underestimate the magnitude of errors, especially fatal ones. ${ }^{52}$

\section{Visibility of Registry Data and Communicating Research Findings}

PGRs may serve as an important channel for participants to learn about the results of their own research initiatives as well as those of other clinical trials for the condition of interest. Although most participants ( $>90 \%$ ) would like to receive a summary of the results of trials in which they participate, less than 10 percent actually receive one. ${ }^{53-55}$ PGRs may serve as a vehicle to provide feedback to and enable dialogue with participants about ongoing and completed research. ${ }^{54} \mathrm{~A}$ recent report from the Institute of Medicine on this topic recommended sharing results with participants in clinical trials and delineated a role for advocacy organizations with regard to 
requiring data sharing plans as part of the criteria for considering a proposal for funding, also providing guidance and education to their members on this topic. ${ }^{56}$

It may also be more common in PGRs than traditional registries that participants gain a greater degree of visibility into the data collected in the registry. In traditional registries, data may be collected directly from clinicians and/or participants, without the participant having access to their own data or knowing how their data compares to other registry participants. Though federal law now gives Americans the right to request a copy of their health record data, ${ }^{57}$ such data are likely to be provided in a form that is not useful to most people, such as a comma-separated variable (CSV) file lacking an explanatory codebook or a portable document format (PDF) that cannot be imported into a more consumer-friendly application. Because most traditional registries have a research emphasis, the data stored may not be available to other allied healthcare professionals on the participant's multidisciplinary team (particularly if they are community-based) or their primary care provider if they did not have a role in providing the data to the registry, especially considering that research data and clinical data receive oversight from different regulatory systems and bodies, as discussed in Chapter 7 of Registries for Evaluating Patient Outcomes: A User's Guide. ${ }^{58}$ More recent developments in the learning healthcare system have led to better interaction and interoperability between these systems; however, they may not yet be sufficient to provide seamless data sharing. Also, researchers may not be sure how to deliver these insights back to participants in studies without counseling and/or support.

By contrast, some, though not all, PGRs permit participants to see their own data as they accumulate over time, and a few even provide graphs or other visual displays of the data in order to allow participants to examine their own patterns in their disease course. As compared to other registries, this allows participants to gain insights into the progression and management of their own condition in real time. In PGRs supported by platforms such as PEER, created by the Genetic Alliance $^{59}$ and Private Access, ${ }^{60}$ the participants control sharing, privacy, and access to their data. An individual may choose to allow some or all of their data to be visible to a number of other parties for the purpose of research and/or support services.

\section{Participant-Led Research}

PGRs have been used for participant-led research, where participants themselves plan and engage in research, and encourage other patients to take part in research, including clinical trials, for about 20 years. For example, Sharon Terry (the founder of PXE International) initiated and led the effort to discover the gene for PXE in $1995 .^{61,62}$ Such initiatives provide a challenge to some of the norms of the research enterprise, such as ethical oversight, because in this case the study is "apomediated" (i.e., mediated between equals rather than in a more imbalanced situation of patients and medical researchers). ${ }^{63}$

In 2007, a group of ALS patients began developing their own research protocol when they translated an Italian conference abstract into English and found that a research group was claiming the mood stabilizer lithium carbonate seemed to slow progression of their disease. A patient in Brazil and a caregiver in the United States with a Ph.D. in geology began encouraging other patients to ask their doctors to prescribe lithium off-label and submit their data on a Google 
Spreadsheet. Soon after, the Italian group's findings were published in the Proceedings of the National Academy of Sciences (PNAS) with the provocative title "Lithium delays progression of amyotrophic lateral sclerosis." ${ }^{\circ 4}$ Faced with a bleak prognosis, many patients started taking lithium and reporting their data online.

In response, the PGR PatientsLikeMe upgraded their data capture systems to collect information about dosage, blood levels, side effects, and longitudinal ALSFRS-R. Although the original Italian study was published in the peer-reviewed literature, it was small, with just 16 lithiumtreated participants and 28 controls. By contrast, more than 160 patients started taking lithium off-label and tracked their data online, with many times that number being available as historical controls.

Both patient groups and researchers from PatientsLikeMe conducted their own analyses of the data. The patient group used rudimentary statistics such as Student's T-Test to look at differences in progression rate between self-treating participants and a random sample of control participants, without adjusting for possible differences in the participants who self-treated from those who were not treated with lithium. While methodologically imperfect, the patient group quickly realized that lithium did not seem to be having a strong effect on progression. ${ }^{65} \mathrm{~A}$ more nuanced statistical analysis by researchers at PatientsLikeMe used a carefully matched set of control participants and published their findings in the peer-reviewed literature along with a deidentified copy of the dataset to allow replication. Like the patient group, they could find no effect of lithium on progression. ${ }^{66}$ While the patient-led study generated a great deal of interest outside the field, traditional academic researchers still went ahead with a number of randomized double-blind placebo controlled trials. Although the data collected through PGRs can be rapid and a powerful means of exploring new hypotheses, today traditional methods of generating evidence such as randomized controlled trials (RCTs) remain the preferred means of accepting or rejecting specified hypotheses around the efficacy and safety of new medicines. In this example, none of those randomized double-blind placebo controlled trials found a beneficial effect of lithium on disease progression, or the trials were halted for futility. ${ }^{67}$

Genomera, a Silicon Valley start-up, is an example of newer platforms that are giving individuals the tools to take participant-led research one step further. While still in a "private-beta" mode, Genomera allows individuals to set up trials to address any research question, with a particular focus on genetic associations. With the help of the software, which provides the necessary background information and statistical support, lay members of the public may conduct their own studies. The resulting data is returned to the community along with the results. ${ }^{68}$ This type of participant led research is new and heterogeneous. Even though these studies are not typically associated with an institution such as a university or government agency, many engage in Institutional Review Board (IRB) review, and these topics are beginning to receive discussion in the medical literature. ${ }^{69}$ All of the citizen-led studies and platforms described in this chapter have received IRB approvals.

The constant demand from participants in the PXE registry for more than a response of "I don't know" from healthcare providers led to the 2012 PXE Health Challenge, created by a young woman affected by PXE. ${ }^{70}$ The Health Challenge focused on the impact of lifestyle on health, 
which responded to the desire of individuals from the community who wanted to improve their baseline health. It was very simple in that it allowed open enrollment, anyone could join at any time, and each month PXE International provided a simple 'challenge' in video and written form. Examples include: parking farther away from a destination, increasing the number of stairs walked each day, and sharing a healthy recipe on Facebook and using one as well. Participants completed an online survey each month to report on their progress. A small study showed a possible improvement in measures such as body mass index, cholesterol, and blood pressure as reported by the participants. ${ }^{71}$

\section{Support of Development of Treatment Guidelines}

PGRs may be able to help generate evidence to support the development of treatment guidelines, especially for diseases or conditions lacking existing treatments approved for that condition. The PXE International Registry has followed 4121 people affected by PXE for 20 years. During this time, the foundation has assessed characteristics of the disease through two natural history studies. These studies, comprising a total of 1079 participants, collected self-reported signs and symptoms, including specific information about general health and physiological measures of skin, eye, gastrointestinal, cardiac, and vascular disease. Data were validated in two ways: (1) medical records of a randomly chosen 10-percent sample of the individuals in the first study were examined and found to correlate well with the self-reported information; and (2) a random sample of 60 nonresponders who were contacted and participated in a telephone survey reported a similar spectrum of disease diversity and severity to that reported by the responders.

The PXE International PGR was able to generate hypotheses that with further testing changed standards of practice for the condition including recommendations about: (1) recognizing a retinal bleed in a timely manner to allow for more treatment options, (2) seeking help from an ophthalmologist at the first sign of a retinal bleed, which preserves sight for a longer period of time, (3) recognizing that mineralization in breast tissue is a typical PXE manifestation and biopsy is not needed, since all women with PXE have breast tissue mineralization, ${ }^{25}$ (4) advising that placental calcification is part of PXE and does not affect the fetus, ${ }^{27}$ and (5) advising that men with PXE and testicular microlithiasis do not have to be monitored for testicular cancer. ${ }^{72}$ This case example of translating the findings from a PGR for a rare disease into care coordination and practice guidelines has become a model for other diseases. ${ }^{19,}$, 73-84

\section{Planning and Design Considerations}

The planning and design of a PGR may be a complex enterprise. Much of the information on planning and design contained in the User's Guide is relevant for PGRs. ${ }^{58}$ However, PGRs must also address some unique considerations, particularly related to business models, governance, ethics and informed consent, as discussed below.

\section{Business Models}

PGRs are heterogeneous as to the business model they use and include both not-for-profit and for-profit models. PXE International, a disease advocacy organization for people affected by PXE that also founded and manages the PXE International Registry, is a nonprofit organization, 
which was started with seed money from small private donors, and has been able to expand its funding base with additional support from Yardi and the Helmsley Foundation, as well as the National Institutes of Health (NIH) for research meetings and specific studies. ${ }^{85}$ In contrast, the Lupus Clinical Trials Consortium is largely funded by a single private donor. ${ }^{86}$

A different funding model is represented by the American BRCA Outcomes and Utilization of Testing (ABOUT) network, which is partly financed through the Aetna Foundation, a national health insurance provider in the United States, which also serves as the main recruiter of patients to the PGR. ${ }^{87}$ Twenty Patient-Powered Research Networks (PPRNs) (not all of which are PGRs - most are not) now receive financial support from PCORI, a nonprofit organization funded by the United States government, and are now in the second phase of funding to continue to build infrastructure, streamline processes, and conduct research studies as part of PCORnet. ${ }^{88}$

PatientsLikeMe is a for profit company with a unique business model. While participation is free and no advertising is accepted, it supports its work through the sale of de-identified patient data to pharmaceutical and other companies. The policy is clearly described on the website to ensure transparency, and purchasers of its data sign contracts that prevent them from "re-identifying” or otherwise attempting to match data to specific patients. ${ }^{89}$

\section{Governance}

The role of patients and participants in governance of PGRs includes being involved directly in the PGR's leadership and decision-making as well as working to identify and prioritize research topics, often in collaboration with researchers. ${ }^{90}$ PCORnet, the network funded by PCORI that includes the PPRNs, advocates for meaningful patient and participant involvement in PGR governance as critical to the success of its PPRNs, recognizing that governance models will need to "respect the structure, history and culture of the PGRs" with each being at different points in the setup process of their organization.

Governance structures for PGRs may include advisory boards composed of participants and, in some cases, scientific advisors. Advisory boards and the general principles related to governance of a patient registry are discussed in Chapter 2 of the User's Guide. ${ }^{58}$ Specific governance challenges relevant to PGRs include, among others, funding sustainability, privacy and confidentiality (see section "Participant Privacy and Confidentiality"), ownership of data and any tissue samples (if applicable), outreach to identify and enroll interested participants, research prioritization, collaborations with outside researchers, and dissemination and return of results to participants.

\section{Ethics and Informed Consent}

As with traditional registries, participants need to be informed clearly and transparently about the risks and benefits of research participation and obtain permissions for research that respect human rights. Chapter 7 of the User's Guide ${ }^{58}$ describes the ethical framework applicable to patient registries collecting data in the United States, including when registries are considered human subjects research and when ethical review is required. 
Best practices and the use and acceptability of IRB reliance relationships in the context of PGRs is encouraged by PCORI's PCORnet. ${ }^{90}$ Potential risks to address include the risks of exposing the participant's identity during data gathering, data dissemination, and publication, quoting directly from online conversations and stories, the ability to identify patients even with deidentified information using data linkage of several sources, and issues with recruiting vulnerable populations such as children. ${ }^{91}$ PGRs and researchers need to be aware of potential ramifications of privacy breaches, the potential harms of such activities, and how to address them. Steps to ensure data security and privacy in collecting, storing, and sharing patients' data are paramount in building and sustaining patient trust and encouraging patients' participation in research.

PGRs may also obtain informed consent from participants, depending on the purpose and design of the registry. In many cases, the informed consent may be obtained electronically, via the registry website. The section on 'Documentation and Format of Consent' in Chapter 8 of the User's Guide $^{58}$ provides an in-depth discussion of the considerations related to electronic consent.

\section{Challenges and Limitations}

PGRs, much like traditional registries, have the potential to support the development of new therapies, facilitate further research, and shape the health care decisions made by patients and providers. PGRs also must address many of the same questions with regard to implementation as investigator-driven registries. In addition to the challenges faced by traditional registries, PGRs face the hurdle of gaining the respect of the medical research community as credible, scientific endeavors without having the underpinnings of academic affiliations and infrastructure. Concerns the PGRs must address, similar to traditional researcher-led registries include participant privacy and confidentiality of health data, potential for bias, validation of the participant population, and data quality.

\section{Participant Privacy and Confidentiality}

A fundamental question is how to allow access to the data available in PGRs for the broader benefit of other patients while allowing participants to retain a sense of control over their health information and ensuring appropriate privacy and confidentiality is maintained. Allowing researchers to contact PGR participants or otherwise request access to PGR data is one consideration. Members of PGRs do not always have an a priori expectation that they will become research participants and may not welcome researchers' contact unless clearly indicated by the PGR policies. ${ }^{91}$

The previously mentioned PEER online system is an example of a technology platform designed to further development of PGRs that has arisen in large part out of concerns for ensuring participants' free choice of how, when, and who to allow access to their data. Participants are given the choice of whether to give disease advocacy and support groups, researchers, users of data analysis platforms, and selected research networks access to their own data by setting preferences to "Allow, Deny, or Ask Me" for each, and participants may change these preferences over time. Data that may be shared includes clinical data, biospecimens, and soon 
direct uploading of EHR data. ${ }^{59}$ The PEER platform is now the technology foundation for the Community Engaged Network for All (or CENA PPRN), funded through PCORnet, which is unique even among the PPRNs for its inclusion of eleven disease advocacy organizations along with university researchers from University of California, San Francisco (UCSF) and UC Davis. $^{92}$

In focus groups during which patients and caregivers with neurological disorders were asked about PGRs, patients were concerned about privacy, which was a strong predictor of their willingness to participate in a PGR. ${ }^{93}$ Privacy concerns include the risk of data falling into the hands of employers and health insurance companies. ${ }^{93}$ Patients with more severe conditions like ALS appeared to have less reluctance about sharing their medical information, which may reflect a sense of urgency for research to develop meaningful treatment options in these more severely affected patients. ${ }^{93}$

\section{Potential for Bias}

Bias is a concern in all observational research and is discussed at length in the section on 'Internal and External Validity' in Chapter 3 of the User's Guide. ${ }^{58}$ The areas of particular concern regarding bias depend on the purpose and design of the registry, whether it is a PGR or a traditional registry. For PGRs, the possibility that participants enrolled in such registries are not representative of the patient population as a whole is of particular concern, yet this selective enrollment may or may not affect the outcome under study. ${ }^{13,94}$ There is a concern that PGR registry participants may be those individuals who feel the strongest - positively or negatively about a particular treatment, thus producing a "reporting bias." 95 To the extent that registry participants may participate in more than one registry or study, some individuals may be overrepresented in reports of multiple studies, especially in the context of a rare disease, though this is more difficult to quantify and also occurs in traditional registries and clinical studies.

\section{Validation of Participant Population}

Unlike other types of observational studies, PGRs often face questions about validation of the diagnoses reported by enrolled participants. Many PGRs allow participants to enroll directly and report that they have received a particular diagnosis. Without verification of that diagnosis by a health care provider or through medical record review, the medical research community may view the data skeptically. Some PGRs require verification of diagnosis from health care providers; in the AAAS survey, 46 percent of registries required such verification. Other PGRs may validate the diagnosis for a sample of participants using medical record review or contact with health care providers. Increasingly, linkage to EHR can be a useful tool for validating both diagnoses and other information (e.g., laboratory tests, exam results) reported by participants, resulting in greater confidence in the validity of the registry data. Use of smartphone technology to report symptoms or to transmit photos can also aid verification of diagnosis. Data being gathered by 23andMe shows a tremendous concordance between self-reported and clinically reported health information. ${ }^{96}$ 


\section{Data Quality and Data Standards}

PGRs face challenges related to data quality, particularly with regard to lack of uniform data collection, insufficient data quality checks, and lack of validation through medical records as primary source documents. However, these may be more reflective of the nature of self-reported data than the fact that these registries are participant-generated.

One example of lack of uniformity is that PGRs may offer participants the opportunity to enter data at time points of their own choosing rather than fixed reporting intervals as used in clinical trials (e.g., baseline measurement with repeated visits at 30 day intervals). A concern with this approach is that there may be nonrandom drivers of data entry, such as a tendency for participants to enter data more frequently when they experience a change in symptoms, particularly a worsening of their condition, resulting in an inaccurate depiction of their disease severity. It also may be that health records face the same bias since people may only visit the doctor when symptoms are worse and do not then record with the same detail during the quieter periods. The increase in the use of mobile health applications may soon provide a rich source of objective data otherwise unobtainable. ${ }^{97}$ Further, most registry-based studies, PGR or traditional, face this challenge since visits are generally not paid for and occur as they do in natural life and not in forced intervals of say thirty days unless that reflects traditional medical care.

Excessive data quality checks in web-based data entry systems may frustrate patients, just as they do physicians, and lead to more missing data rather than higher quality data. ${ }^{98}$ Certain types of data may also be more prone to errors when reported directly by participants; examples include results for laboratory tests, genetic test results, and data that include units, such as HbA1c. Additional variability can be introduced by lack of uniform measurement tools, e.g., reporting weight from a typical home scale. Lack of verification of data through medical record review or contact with health care providers can introduce concerns about the data validity and quality.

The use of common data elements (CDEs) when developing a new PGR has the potential to improve the quality of the data collected in the registry and support future efforts to aggregate or compare data across studies. While CDEs have not been developed for all condition areas, the availability of CDEs is increasing as more organizations fund efforts to develop data standards. For example, the National Institute of Neurological Disorders and Stroke (NINDS) maintains a repository of several hundred data elements and definitions related to neurological conditions. ${ }^{99}$ Another example is the list of CDEs developed by the Office of Rare Disease Research (ORDR) at the NIH for use in rare disease registries. ${ }^{100} \mathrm{~A}$ useful starting place for information about CDEs is the CDE portal maintained by the National Library of Medicine; ${ }^{101}$ the User's Guide also discusses data element selection and CDEs in Chapter $4 .{ }^{58}$ While not all of the available CDEs may be appropriate for inclusion within PGRs, these initiatives can provide useful tools to support the development of a new registry. 


\section{Conclusion}

As clinical research strives to be patient-centered, PGRs offer an existing infrastructure to support new research as well as tools to reach patients and build communities. These offer a trusted community, often with relative ease of access and simplicity. Results from PGRs can generate evidence to help guide patients' and providers' treatment decisions, as well as generate new research questions meriting rigorous study approaches. The increasing implementation of best practices and evaluation criteria begins to address prior questions of scientific credibility.

Efforts are needed to inform the medical research community about the potential of PGRs and participant-generated research to provide relevant and timely evidence that can complement findings from traditional studies. Such efforts may be assisted through sharing of case studies that highlight successful uses of PGRs and through dissemination activities related to best practices. Recent and highly visible public efforts, such as the creation of PCORI's PPRN, provide further opportunity for increasing awareness and acceptance of the potential contributions of PGRs. 


\section{References}

1. PRIDE

Study. http://www.pridestudy.org/TheStudy. html. Accessed January 10, 2017.

2. Direct-to-Patient Registry and Other PatientCentric Designs. In: Gliklich RE, Dreyer NA, Leavy MB, Christian JB (eds). Increasing Focus on the Patient in Patient Registries. (Prepared by L\&M Policy Research and QuintilesIMS under Contract No. HHSA290201400004C.) Rockville, MD: Agency for Healthcare Research and Quality. Forthcoming 2017.

3. Terry SF. Learning Genetics. Health Aff (Millwood). 2003 Sep-Oct;22(5):166-71. PMID: 14515892.

4. Moore's Law. http://www.mooreslaw.org/. Accessed January 10, 2017.

5. Valdes I, Kibbe D, Tolleson G, et al. Metcalfe's law predicts reduced power of Electronic Medical Record software. AMIA Annu Symp Proc. 2003:1038. PMID: 14728541. PMCID: Pmc1480183. Epub 2004/01/20. eng.

6. Friend SH, Norman TC. Metcalfe's law and the biology information commons. Nat Biotechnol. 2013 Apr;31(4):297-303. PMID: 23563423. Epub 2013/04/09. eng.

7. American Association for the Advancement of Science: Social Networking and Online Health Communities: Identifying and Describing Patient-Generated Registries. http://www.aaas.org/page/socialnetworking-and-online-health-communitiesidentifying-and-describing-patientgenerated. Accessed January 10, 2017.

8. Merideth MA, Gordon LB, Clauss S, et al. Phenotype and course of Hutchinson-Gilford progeria syndrome. N Engl J Med. 2008 Feb 7;358(6):592-604. PMID: 18256394.

PMCID: 2940940.

9. Gordon LB, Rothman FG, Lopez-Otin C, et al. Progeria: a paradigm for translational medicine. Cell. 2014 Jan 30;156(3):400-7. PMID: 24485450. Epub 2014/02/04. eng.

10. Ullrich NJ, Gordon LB. Hutchinson-Gilford progeria syndrome. Handb Clin Neurol. 2015;132:249-64.

11. NARCOMS Multiple Sclerosis Patient Registry. http://narcoms.org/. Accessed January 10, 2017.

12. iConquerMS. https://www.iconquerms.org/. Accessed January 10, 2017.

13. Dreyer NA, Blackburn S, Mt-Isa S, et al. Direct-to-patient research: piloting a new approach to understanding drug safety during pregnancy. JMIR Public Health and Surveillance. 2015;2(1):e22.

14. Lao W-L, Malone DC, Armstrong EP, et al. Effect of adjustable gastric banding on quality of life and weight loss in the Helping Evaluate Reduction in Obesity (HERO) registry study: 2-year analysis. Curr Med Res Opin. 2015;31(8):1451-60.

15. Freudheim M. Tool in Cystic Fibrosis Fight: A Registry. The New York Times. 2009 December 21, 2009.

16. 50 Years of Research, Hope, Progress. http://www.cff.org. Accessed January 10, 2017.

17. Rangel V, Martin AS, Peay HL. DuchenneConnect Registry Report. PLoS Curr. 2012;4:Rrn1309. PMID: 22453902. PMCID: Pmc3299489. Epub 2012/03/29. eng.

18. Wang RT, Silverstein Fadlon CA, Ulm JW, et al. Online self-report data for duchenne muscular dystrophy confirms natural history and can be used to assess for therapeutic benefits. PLoS Curr. 2014;6. PMID: 25635234. PMCID: Pmc4207635. Epub 2015/01/31. eng.

19. Terry SF, Terry PF, Rauen KA, et al. Advocacy groups as research organizations: 
the PXE International example. Nat Rev Genet. 2007 Feb;8(2):157-64. PMID: 17230202. eng.

20. Phillips AG, Hongaard-Andersen P, Moscicki RA, et al. Proceedings of the 2013 CINP summit: innovative partnerships to accelerate CNS drug discovery for improved patient care. Int J Neuropsychopharmacol. 2015 Feb;18(3). PMID: 25542690. Epub 2014/12/30. eng.

21. Gordon LB, Massaro J, D'Agostino RB, Sr., et al. Impact of farnesylation inhibitors on survival in Hutchinson-Gilford progeria syndrome. Circulation. $2014 \mathrm{Jul}$ 1;130(1):27-34. PMID: 24795390. PMCID: Pmc4082404. Epub 2014/05/06. eng.

22. Turner MR, Wicks P, Brownstein CA, et al. Concordance between site of onset and limb dominance in amyotrophic lateral sclerosis. J Neurol Neurosurg Psychiatry. 2011 Aug;82(8):853-4. PMID: 20562391. Epub 2010/06/22. eng.

23. Mullins CD, Vandigo J, Zheng Z, et al. Patient-centeredness in the design of clinical trials. Value Health. 2014 Jun;17(4):471-5. PMID: 24969009. Epub 2014/06/28. eng.

24. Bercovitch L, Martin L, Chassaing N, et al. Acquired pseudoxanthoma elasticum presenting after liver transplantation. J Am Acad Dermatol. 2011 May;64(5):873-8. PMID: 21397982. PMCID: Pmc3078966. Epub 2011/03/15. eng.

25. Bercovitch L, Schepps B, Koelliker S, et al. Mammographic findings in pseudoxanthoma elasticum. J Am Acad Dermatol. 2003 Mar;48(3):359-66. PMID: 12637915.

26. Bercovitch L, LeRoux T, Terry SF, et al. Pregnancy and obstetrical outcomes in pseudoxanthoma elasticum. Br J Dermatol. 2004 Nov;151(5):1011-8.

27. Gheduzzi D, Taparelli F, Quaglino D, Jr., et al. The placenta in pseudoxanthoma elasticum: clinical, structural and immunochemical study. Placenta. 2001 Jul;22(6):580-90. PMID: 11440547.
28. U.S. Food and Drug Administration. Guidance for Industry. Patient-Reported Outcome Measures: Use in Medical Product Development to Support Labeling Claims. Federal Register 2009; 74(35). p. 65132133.

29. Cedarbaum JM, Stambler N, Malta E, et al. The ALSFRS-R: a revised ALS functional rating scale that incorporates assessments of respiratory function. BDNF ALS Study Group (Phase III). J Neurol Sci. 1999 Oct 31;169(1-2):13-21. PMID: 10540002.

30. Wicks P. Commentary: Measuring what matters: the case for patient generated PROMS. BMJ. 2015;350:h54. PMID: 25670198.

31. Wicks P. Measuring function in advanced ALS: validation of ALSFRS-EX extension items. European Journal of Neurology 2009;16(3):6.

32. Brady CB, Trevor KT, Stein TD, et al. The Department of Veterans Affairs Biorepository Brain Bank: a national resource for amyotrophic lateral sclerosis research. Amyotroph Lateral Scler Frontotemporal Degener. 2013 Dec;14(78):591-7. PMID: 23971854. Epub 2013/08/27. eng.

33. Abdulla S, Vielhaber S, Korner S, et al. Validation of the German version of the extended ALS functional rating scale as a patient-reported outcome measure. J Neurol. 2013 Sep;260(9):2242-55. PMID: 23736973. Epub 2013/06/06. eng.

34. Burke LB, Kennedy DL, Miskala PH, et al. The use of patient-reported outcome measures in the evaluation of medical products for regulatory approval. Clin Pharmacol Ther. 2008 Aug;84(2):281-3. PMID: 18580868. Epub 2008/06/27. eng.

35. Tran VT, Harrington M, Montori VM, et al. Adaptation and validation of the Treatment Burden Questionnaire (TBQ) in English using an internet platform. BMC Med. 2014;12:109. PMID: 24989988. PMCID: Pmc4098922. Epub 2014/07/06. eng 
36. Rothman M, Gnanaskathy A, Wicks P, et al. Can we use social media to support content validity of patient-reported outcome instruments in medical product development? Value Health. 2015 Jan;18(1):1-4. PMID: 25595228. Epub 2015/01/18. eng.

37. Cascade E, Marr P, Winslow M, et al. Conducting research on the Internet: medical record data integration with patientreported outcomes. J Med Internet Res. 2012;14(5):e137. PMID: 23060427. PMCID: Pmc3510750. Epub 2012/10/13. eng.

38. McCarrier K, Wicks P. Online social networks-based qualitative research to identify participant-relevant concepts in chronic lymphocytic leukemia. Health. 2014;4(8).

39. BioMarin Pharmaceutical. A Phase $1 / 2$ Open-Label Dose-Escalation Study to Evaluate Safety, Tolerability, Pharmacokinetics, and Efficacy of Intracerebroventricular BMN 190 in Patients With Late-Infantile Neuronal Ceroid Lipofuscinosis (CLN2) Disease. In: ClinicalTrials.gov. Bethesda (MD): National Library of Medicine (US). https://clinicaltrials.gov/show/NCT01 907087. Accessed January 10, 2017.

40. de Blieck EA, Augustine EF, Marshall FJ, et al. Methodology of clinical research in rare diseases: development of a research program in juvenile neuronal ceroid lipofuscinosis (JNCL) via creation of a patient registry and collaboration with patient advocates. Contemp Clin Trials. 2013;35(2):48-54.

41. What We Mean by Engagement: Engagement in Research. http://www.pcori.org/fundingopportunities/what-we-mean-engagement. Accessed January 10, 2017.

42. Frank L, Basch E, Selby JV. The PCORI Perspective on Patient-Centered Outcomes Research. JAMA. 2014;312(15):1513-4.
43. Office of the Press Secretary, The White House. FACT SHEET: President Obama's Precision Medicine Initiative. 2015. https://www.whitehouse.gov/the-pressoffice/2015/01/30/fact-sheet-presidentobama-s-precision-medicine-initiative. Accessed January 10, 2017.

44. The White House: The Precision Medicine Initiative https://www.whitehouse.gov/preci sion-medicine. Accessed January 10, 2017.

45. Perfetto EM, Burke LB, Oehrlein EM, et al. Patient-Focused Drug Development: A New Direction for Collaboration. Med Care. 2015;53(1):9-17.

46. Peay HL, Hollin I, Fischer R, et al. A community-engaged approach to quantifying caregiver preferences for the benefits and risks of emerging therapies for Duchenne muscular dystrophy. Clin Ther. 2014 May;36(5):624-37. PMID: 24852596. Epub 2014/05/24. eng.

47. Allison M. Can web 2.0 reboot clinical trials? Nat Biotechnol. 2009 Oct;27(10):895-902. PMID: 19816442. Epub 2009/10/10. eng.

48. Reg4All Trials Finder. https://www.reg4all.org/. Accessed January 10, 2017.

49. Fox Trial Finder. https://foxtrialfinder.michaeljfox.org /. Accessed January 10, 2017.

50. PatientsLikeMe. https://www.patientslikeme .com/. Accessed January 10, 2017.

51. The Empowered Patient Coalition. http://empoweredpatientcoalition. org/. Accessed January 10, 2017. 
52. Southwick FS, Cranley NM, Hallisy JA. A patient-initiated voluntary online survey of adverse medical events: the perspective of 696 injured patients and families. BMJ Qual Saf. 2015 Oct;24(10):620-9.

53. Cox K, Moghaddam N, Bird L, et al. Feedback of trial results to participants: a survey of clinicians' and patients' attitudes and experiences. Eur J Oncol Nurs. 2011 Apr;15(2):124-9. PMID: 20674498. PMCID: 3078333.

54. Partridge AH, Winer EP. Sharing study results with trial participants: time for action. J Clin Oncol. 2009 Feb 20;27(6):838-9. PMID: 19164202. Epub 2009/01/24. eng.

55. Getz K, Hallinan Z, Simmons D, et al. Meeting the obligation to communicate clinical trial results to study volunteers. Expert Rev Clin Pharmacol. 2012 Mar;5(2):149-56. PMID: 22390557.

56. Institute of Medicine. Sharing Clinical Trial Data: Maximizing Benefits, Minimizing Risk. http://www.nap.edu/catalog/18998/sha ring-clinical-trial-data-maximizing-benefitsminimizing-risk. Accessed January 10, 2017.

57. Fioriglio G, Szolovits P. Copy fees and patients' rights to obtain a copy of their medical records: from law to reality. AMIA Annu Symp Proc. 2005:251-5. PMID: 16779040. PMCID: 1560890.

58. Gliklich R, Dreyer N, Leavy M, eds. Registries for Evaluating Patient Outcomes: A User's Guide. Third edition. Two volumes. (Prepared by the Outcome DEcIDE Center [Outcome Sciences, Inc., a Quintiles company] under Contract No. 290 200500351 TO7.) AHRQ Publication No. 13(14)-EHC111. Rockville, MD: Agency for Healthcare Research and Quality. April 2014. http://www.effectivehealthcare.ahrq.g ov/registries-guide-3.cfm. Accessed January 10, 2017.

59. Platform for Engaging Everyone Responsibly. http://www.geneticalliance.org /programs/biotrust/peer. Accessed January 10, 2017.

60. Private Access, Inc. | Your solution for controlling who sees your personal health information. https://www.privateaccess.info/ . Accessed January 10, 2017.

61. Bergen AA, Plomp AS, Schuurman EJ, et al. Mutations in ABCC6 cause pseudoxanthoma elasticum. Nat Genet. 2000 Jun;25(2):228-31.

62. Le Saux O, Urban Z, Tschuch C, et al. Mutations in a gene encoding an $\mathrm{ABC}$ transporter cause pseudoxanthoma elasticum. Nat Genet. 2000 Jun;25(2):223-7.

63. O'Connor D. The apomediated world: regulating research when social media has changed research. J Law Med Ethics. 2013 Summer;41(2):470-83. PMID: 23802898.

64. Fornai F, Longone P, Cafaro L, et al. Lithium delays progression of amyotrophic lateral sclerosis. Proc Natl Acad Sci U S A. 2008 Feb 12;105(6):2052-7. PMID: 18250315. PMCID: 2538879.

65. Felzer K, Macedo H. The Lithium ALS Worldwide Study: Six Month Update. 2008.

66. Wicks P, Vaughan TE, Massagli MP, et al. Accelerated clinical discovery using selfreported patient data collected online and a patient-matching algorithm. Nat Biotechnol. 2011 May;29(5):411-4. PMID: 21516084. Epub 2011/04/26. eng.

67. Armon C. Is the lithium-for-ALS genie back in the bottle?: Not quite. Neurology. 2010 Aug 17;75(7):586-7. PMID: 20702793.

68. Swan M. Crowdsourced health research studies: an important emerging complement to clinical trials in the public health research ecosystem. J Med Internet Res. 2012;14(2):e46. PMID: 22397809. PMCID: 3376509. Epub 2012/03/09. eng. 
69. Vayena E, Tasioulas J. Adapting Standards: Ethical Oversight of Participant-Led Health Research. PLoS Med. 2013;10(3):e1001402. Epub 2013 Mar 12.

70. PXE International: Health Challenge. https://www.pxe.org/aboutpseudoxanthoma-elasticum/pxeinternational-health-challenge. Accessed January 10, 2017.

71. PXE International Health Challenge, Poster Presentation at the PXE Biennial Research Meeting, Bethesda, MD. September 22, 2014.

72. Bercovitch RS, Januario JA, Terry SF, et al. Testicular microlithiasis in association with pseudoxanthoma elasticum. Radiology. 2005 Nov;237(2):550-4. PMID: 16244264.

73. Terry SF, Davidson ME. Empowering the public to be informed consumers of genetic technologies and services. Community Genet. 2000;3(3):148-50. PMID: 11831266.

74. Terry SF, Davidson, M.E. Meeting the Needs of Affected Individuals in the New Genetics Age. Exceptional Parent. 2000.

75. Lin AE, Terry SF, Lerner B, et al. Participation by clinical geneticists in genetic advocacy groups. Am J Med Genet A. 2003 May 15;119(1):89-92. PMID: 12707968 .

76. Beskow LM, Botkin JR, Daly M, et al. Ethical issues in identifying and recruiting participants for familial genetic research. Am J Med Genet A. 2004 Nov 1;130(4):424-31. PMID: 15455364.

77. Terry SF, Christensen K, Herrick E, et al. Affected Individuals' Perceptions of their Healthcare Providers' Knowledge about Genetic Services. [Paper]. In press 2006.

78. Haga SB, Terry SF. Ensuring the safe use of genomic medicine in children. Clin Pediatr (Phila). 2009 Sep;48(7):703-8. PMID: 19448129. Epub 2009/05/19. eng.
79. Horn EJ, Edwards K, Terry SF. Engaging research participants and building trust. Genet Test Mol Biomarkers. 2011 Dec;15(12):839-40. PMID: 22181985. Epub 2011/12/21. eng.

80. Terry SF, Horn EJ, Scott J, et al. Genetic Alliance Registry and BioBank: a novel disease advocacy-driven research solution. Personalized Medicine. 2011;8(2):207-13.

81. Kaye J, Curren L, Anderson N, et al. From patients to partners: participant-centric initiatives in biomedical research. Nat Rev Genet. 2012 May;13(5):371-6. PMID: 22473380. Epub 2012/04/05. eng.

82. Landy DC, Brinich MA, Colten ME, et al. How disease advocacy organizations participate in clinical research: a survey of genetic organizations. Genet Med. 2012 Feb;14(2):223-8. PMID: 22261756. Epub 2012/01/21. eng.

83. Terry SF. Disease advocacy organizations catalyze translational research. Front Genet. 2013;4:101. PMID: 23761807. PMCID: 3671180 .

84. Uitto J, Varadi A, Bercovitch L, et al. Pseudoxanthoma elasticum: progress in research toward treatment: summary of the 2012 PXE international research meeting. J Invest Dermatol. 2013 Jun;133(6):1444-9. PMID: 23673496. Epub 2013/05/16. eng.

85. PXE International. https://www.pxe.org/about-pxeinternational/history-of-pxe-international. Accessed January 10, 2017.

86. The Lupus Clinical Trials Consortium. http://www.lupusclinicaltrials.o rg/Pages/Default.aspx. Accessed January 10, 2017.

87. Armstrong J, Toscano M, Kotchko N, et al. American BRCA Outcomes and Utilization of Testing (ABOUT) Study: A Pragmatic Research Model that Incorporates Personalized Medicine/Patient-Centered Outcomes in a Real World Setting. J Genet Counsel. 2015;24:18-28. 
88. PCORnet: The National Patient-Centered Clinical Research

Network. http://www.pcornet.org/aboutpcornet/. Accessed January 10, 2017.

89. Golosinski M. Health Care and Social Networks: Creating an Online Patient Community. Insead Knowledge: Healthcare [Internet].

2012. http://knowledge.insead.edu/csr/health care/health-care-and-social-networkscreating-an-online-patient-community-2341. Accessed January 10, 2017.

90. Fleurence RL, Beal AC, Sheridan SE, et al. Patient-Powered Research Networks Aim to Improve Patient Care and Health Research. Health Affairs. 2014;33(7):1212-9.

91. CIRE | Current Issues in Research Ethics: Privacy and Confidentiality. http:/ccnmtl.columbia.edu/ projects/cire/pac/introduction/index.html. Accessed January 10, 2017.

92. PCORnet: Community-Engaged Network for All (CENA). http://www.pcornet.org/patientpowered-research-networks/pprn18-geneticalliance-inc/. Accessed January 10, 2017.

93. Korngut L, MacKean G, Casselman L, et al. Perspectives on Neurological Patient Registries: A Literature Review and Focus Group Study. BMC Medical Research Methodology. 2013;13:135.

94. Frydman GJ. Participant-driven research: rich opportunities and real risks. J Participat Med. 2009;October(1).
95. Frost J, Okun S, Vaughan T, et al. Patientreported outcomes as a source of evidence in off-label prescribing: analysis of data from PatientsLikeMe. J Med Internet Res. 2011;13(1):e6. PMID: 21252034. PMCID: 3221356. Epub 2011/01/22. eng.

96. Tung JY, Do CB, Hinds DA, et al. Efficient replication of over 180 genetic associations with self-reported medical data. PLoS One. 2011;6(8):e23473.

97. Runkle D. Exploring Legal Challenges to Fulfilling the Potential of mHealth in a Safe and Responsible Environment - Workshop Report 1: Regulation. Advancing Science Serving Society; 2015.

98. Mendelsohn AB, Dreyer NA, Mattox PW, et al. Characterization of missing data in clinical registry studies. Therapeutic Innovation \& Regulatory Science. 2014;48(5):574-82.

99. National Institute of Neurological Disorders and Stroke. Common Data Elements. http://www.commondataelements. ninds.nih.gov/\#page=Default. Accessed January 10, 2017.

100. National Center for Advancing Translational Sciences. Common Data Elements. https://ncats.nih.gov/grdr/cdes. Accessed January 10, 2017.

101. National Library of Medicine. Common Data Elements Resource Portal. https://www.nlm.nih.gov/cde/. Accessed January 10, 2017. 